\title{
Como responder a dúvidas clínicas
}

Rodrigues DS, Viegas Dias C, Heleno B.

\begin{abstract}
RESUMO
O conhecimento médico está em permanente construção e estar atualizado é uma tarefa inerente à prática médica. É objetivo deste artigo rever a metodologia para resposta a dúvidas clínicas e propor o modelo dos $5 \mathrm{~A}$ para esse efeito. A obtenção de respostas a perguntas clínicas passa por elaborar adequadamente a pergunta, adquirir a informação necessária, avaliar criticamente essa mesma informação, aplicar a evidência ao nosso contexto e prática e, por fim, atuar de forma a garantir que os doentes recebem os melhores cuidados médicos possíveis. Este exercício deve ser incluído no dia-a-dia da prática médica e, caso preencha critérios de novidade, rigor metodológico e relevância para a prática clínica, divulgado e publicado.
\end{abstract}

Palavras-chave: Medicina baseada na evidência; Questões clínicas; Transmissão do conhecimento; Tomada de decisão; Partilha de decisão informada.

\section{INTRODUÇÃO}

$\mathrm{H}$ á quase 20 anos que nas páginas desta revista se alerta para a necessidade de capacitar os médicos de competências na leitura crítica de artigos para responder a dúvidas clínicas que surgem no dia-a-dia da prática médica. ${ }^{1} \mathrm{O}$ conhecimento está em permanente construção e estar atualizado é uma tarefa inerente à prática médica. ${ }^{2}$

Sackett e colegas sublinhavam a importância das decisões médicas resultarem da integração do melhor conhecimento proveniente da literatura médica com a experiência clínica do médico e os valores, preferências e contexto dos doentes. ${ }^{3} \mathrm{O}$ conceito de medicina baseada na evidência (MBE) - hoje mais generalizada através do conceito de prática clínica baseada na evidência - surgiu para desafiar os médicos a desenvolverem um «ceticismo iluminado, procurando ter consciência da prova científica que sustenta a sua prática e as suas dúvidas clínicas. ${ }^{4} \mathrm{~A}$ MBE promove, através de decisões colaborativas, o encontro entre dois atores (clínico e utente) com diferentes conhecimentos, experiências e perspetivas, mas ambos necessários para atingir o objetivo final de ajudar as pessoas nas escolhas relacionadas com a sua saúde. ${ }^{5}$ Isso pressupõe, no entanto, o conhecimento e entendimento da melhor

Unidade de Medicina Geral e Familiar. NOVA Medical School, Universidade Nova de Lisboa. prova científica proveniente da investigação clínica. ${ }^{4}$ Observamos, no entanto, que nas últimas décadas a produção de literatura médica aumentou para níveis impossíveis de acompanhar. ${ }^{6}$ Atendendo à evolução que a disponibilidade e tipologia de informação médica recentemente teve, é objetivo deste artigo rever a metodologia para resposta a dúvidas clínicas do dia-a-dia da prática clínica.

Como passar então dos conceitos teóricos da MBE para uma prática clínica baseada na evidência? Propõe-se o método dos 5A (Figura 1).

\section{PASSO 1: A PERGUNTA}

A cada doente que vemos aparecem entre duas a cinco perguntas clínicas..$^{7-8}$ Recomendamos que tome nota destas dúvidas clínicas com que fica no final da consulta. Caso contrário, arrisca-se a não resolver essa dúvida que certamente voltará noutra consulta. ${ }^{9}$ Portanto, o primeiro passo da prática clínica baseada na evidência é identificar perguntas que sejam clinicamente relevantes e que tenham impacto na prática clínica.

\section{Perguntas de conhecimento de base}

Ao longo da nossa carreira clínica vamos tendo dúvidas diferentes e, por isso, fazemos diferentes tipos de perguntas. Numa fase inicial, enquanto estudantes de medicina e nos primeiros anos clínicos, a maioria das dúvidas dizem respeito a epidemiologia, mecanismos fi- 


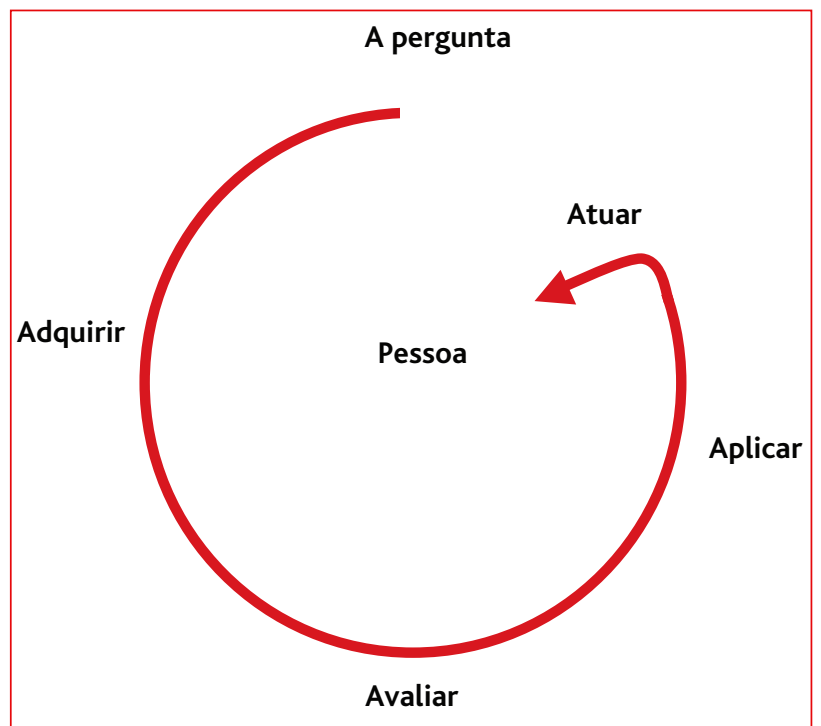

Figura 1. O método dos 5A para uma prática clínica baseada na evidência

Adaptado de: Guyatt G. Users' guides to the medical literature: a manual for evidence-based clinical practice. 3rd ed. (Users Guides to the Medical Literature). McGraw-Hill Education. [Kindle edition.]

siopatológicos das doenças ou ainda opções terapêuticas clássicas. ${ }^{4}$ Estas perguntas são normalmente abrangentes, genéricas e podem ser respondidas recorrendo a livros de texto ou à secção overview de plataformas de apoio à decisão clínica, como o UpToDate, a Dynamed ou o BMJ Best Practice. A estas chamaremos de perguntas de conhecimento de base (Quadro I). Estas perguntas têm habitualmente dois componentes: 1) uma pergunta de raiz (quem, o quê, onde, quando, como, porquê), seguida de um verbo; e 2) uma doença, teste, tratamento ou outro aspeto dos cuidados médicos. ${ }^{10}$

\section{Perguntas de conhecimento orientado por problemas}

À medida que o conhecimento de base solidifica e começamos a ver muitos doentes, começam a aparecer as perguntas de conhecimento orientado por problemas. Estas perguntas visam a obtenção de conhecimento que informa decisões clínicas e leva a recomendações concretas sobre cuidados a serem prestados a doentes. Podemos dividir estas perguntas em perguntas qualitativas e perguntas quantitativas (Quadro II).

Perguntas qualitativas visam descobrir o significado ou compreender um fenómeno. Para isso exploram conteúdos subjetivos como, por exemplo, experiências, sentimentos ou atitudes. Estas perguntas estruturam-se de acordo com o modelo PO, no qual se definem a População [P] e o(s) resultado(s) clínico(s) de interesse (outcome em inglês) [O].

Perguntas quantitativas procuram estimar um fenómeno ou estabelecer relações entre variáveis (e.g., um tratamento e o sucesso terapêutico, um fator de risco e uma doença, o resultado de um teste não invasivo e o resultado de uma biópsia). É mais fácil responder a perguntas que sejam estruturadas e o acrónimo PICO resume alguns dos principais componentes: $\mathrm{P}-\mathrm{a}$ população ou problema de interesse; I - a principal intervenção em estudo, definida de forma genérica, podendo ser uma exposição, um teste diagnóstico, um fator de prognóstico, um tratamento, uma perceção dos doentes, entre outras; $\mathrm{C}$ - uma intervenção ou exposição comparadora; $\mathrm{O}$-o(s) resultado(s) clínico(s) de interesse (outcome em inglês), incluindo o horizonte temporal, se relevante. ${ }^{10}$ No Quadro II exemplificam-se as diferentes estruturas para perguntas de conhecimento orientado por problemas.

Importa sublinhar três aspetos: o primeiro é que mesmo nos anos iniciais do ensino médico é desejável que apareçam perguntas orientadas por problemas. $\mathrm{O}$ segundo é que, da mesma forma, nunca seremos sufi-

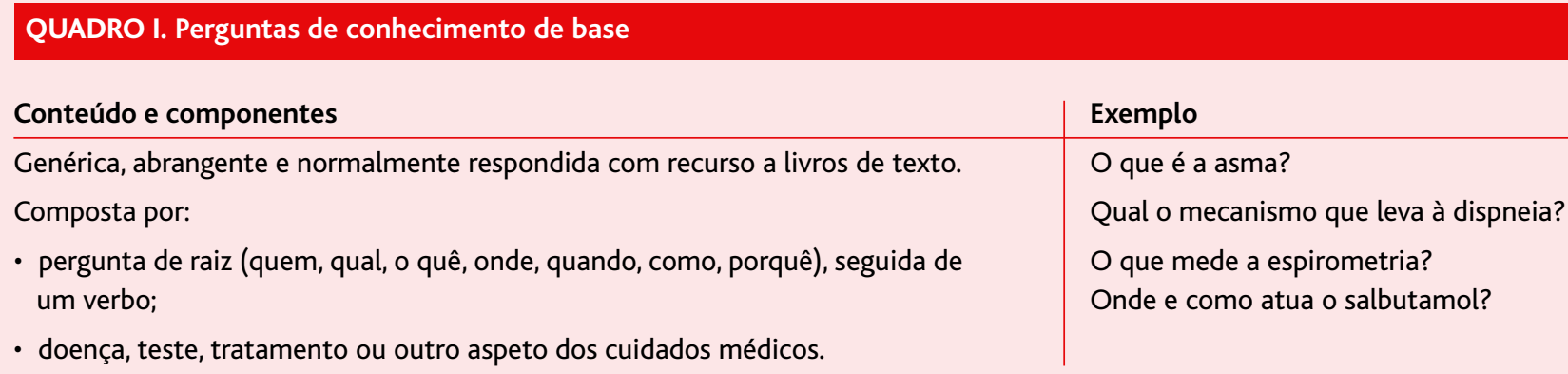

\section{Conteúdo e componentes}

Genérica, abrangente e normalmente respondida com recurso a livros de texto.

Composta por:

- pergunta de raiz (quem, qual, o quê, onde, quando, como, porquê), seguida de um verbo;

- doença, teste, tratamento ou outro aspeto dos cuidados médicos.

Exemplo

O que é a asma?

Qual o mecanismo que leva à dispneia?

O que mede a espirometria?

Onde e como atua o salbutamol? 
QUADRO II. Perguntas de conhecimento orientado por problemas

Perguntas Qualitativas - Modelo PS

Como é que __ $[\mathrm{P}] \_$_ perceciona/experiencia/vivencia/compreende ___ $[\mathrm{S}] \_$?

Ex1: Como é que cuidadores de doentes de Alzheimer vivenciam a institucionalização desses doentes?

Ex2: Como é que mulheres em idade de realizar rastreio de cancro da mama compreendem o conceito de sobrediagnóstico?

Perguntas Quantitativas - Modelo PICO

Tratamento

Em _ _ $[\mathrm{P}] \_$, será que __ $[\mathrm{l}] \_$quando comparada com __ $[\mathrm{C}] \_$leva a __ $[\mathrm{O}] \_$?

Ex: Em pessoas com diabetes tipo 2 será que a toma de aspirina, quando comparada com a não toma, leva a menos eventos cardiovasculares?

\section{Etiologia (risco)}

Em _ $[\mathrm{P}] \ldots$, será que __ $[\mathrm{E}] \_$quando comparada com __ $[\mathrm{C}] \_$leva a __ $[\mathrm{O}] \_$?

Ex: Em mulheres não fumadoras, será que exposição diária passiva a fumo de tabaco, quando comparada com a não exposição, leva a mais casos de neoplasia do pulmão?

Diagnóstico

$\mathrm{Em} \_[\mathrm{P}] \_$, qual a aquidade diagnóstica de _ $[\mathrm{I}] \_$quando comparada com o padrão ouro _[C]__ para a doença __ [O]__? Uma formulação mais simples e frequentemente usada é:

$\mathrm{Em} \_[\mathrm{P}] \_$, será que __ $[\mathrm{I}] \_$consegue diagnosticar __ $[\mathrm{O}] \_$?

Ex1: Em adultos com suspeita de enfarte agudo do miocárdio, será que ECGs seriados, quando comparados com um único ECG inicial, tem maior acuidade diagnóstica para enfarte agudo do miocárdio?

Ex2: Em adultos com dispneia aguda será que uma cintigrafia de ventilação / perfusão consegue detetar tromboembolismo pulmonar?

Prevenção

Em _ _ $[\mathrm{P}] \ldots$, será que __ $[\mathrm{I}] \ldots$ quando comparada com __ $[\mathrm{C}] \_$leva a __ $[\mathrm{O}] \_$?

Ex1: Em adultos fumadores com mais de 55 anos será que uma TAC torácica bianual, quando comparada com não realização da mesma, leva a menor mortalidade?

Prognóstico

Em __ $[\mathrm{P}] \ldots$, será que __ $[\mathrm{I}] \_$quando comparada com __ $[\mathrm{C}] \_$leva a __ $[\mathrm{O}] \_$?

Ex1: Em adultos com mais de 65 anos será que a vacina da gripe, quando comparada com não realização da mesma, leva a menor mortalidade?

cientemente experientes de forma a não ter perguntas de conhecimento de base. O terceiro é que devemos reconhecer, tomar nota e responder às nossas perguntas. Uma pergunta não registada é uma pergunta que fica por responder e é apenas uma questão de tempo até que apareça de novo.

\section{PASSO 2: ADQUIRIR}

Depois de elaborarmos uma pergunta adequada, o passo seguinte é fazer a pesquisa bibliográfica. Hoje em dia temos disponíveis uma série de ferramentas que rapidamente nos disponibilizam informação médica resumida e pré-avaliada. A nossa pesquisa deve então 
incidir primeiro em fontes que já tenham sintetizado e avaliado toda a prova científica relevante para a nossa pergunta.

A Figura 2, adaptada de Alper e Haynes, ${ }^{11}$ sugere uma estrutura de cinco níveis hierárquicos de informação clínica, a pirâmide $5 \mathrm{~S}$ de prática clínica baseada na evidência. Cada nível inclui de forma sistemática todos os níveis inferiores e quanto maior o nível maior o potencial para informar a decisão clínica baseada na melhor prova científica. ${ }^{11}$

No topo da pirâmide está a situação ideal: o sistema de informação clínica baseado na evidência. Um sistema neste contexto é aquele que sumariza e integra toda a prova científica relevante e pertinente sobre determinado problema clínico e apresenta-a dentro do registo clínico eletrónico de um utente no momento adequado. Infelizmente estes sistemas ainda não fazem parte do nosso dia-a-dia, mas provavelmente serão uma realidade para todos nós muito em breve.

Num segundo nível superior na pirâmide 5S estão os Sumários de Apoio

à Decisão Clínica. Estas ferramentas

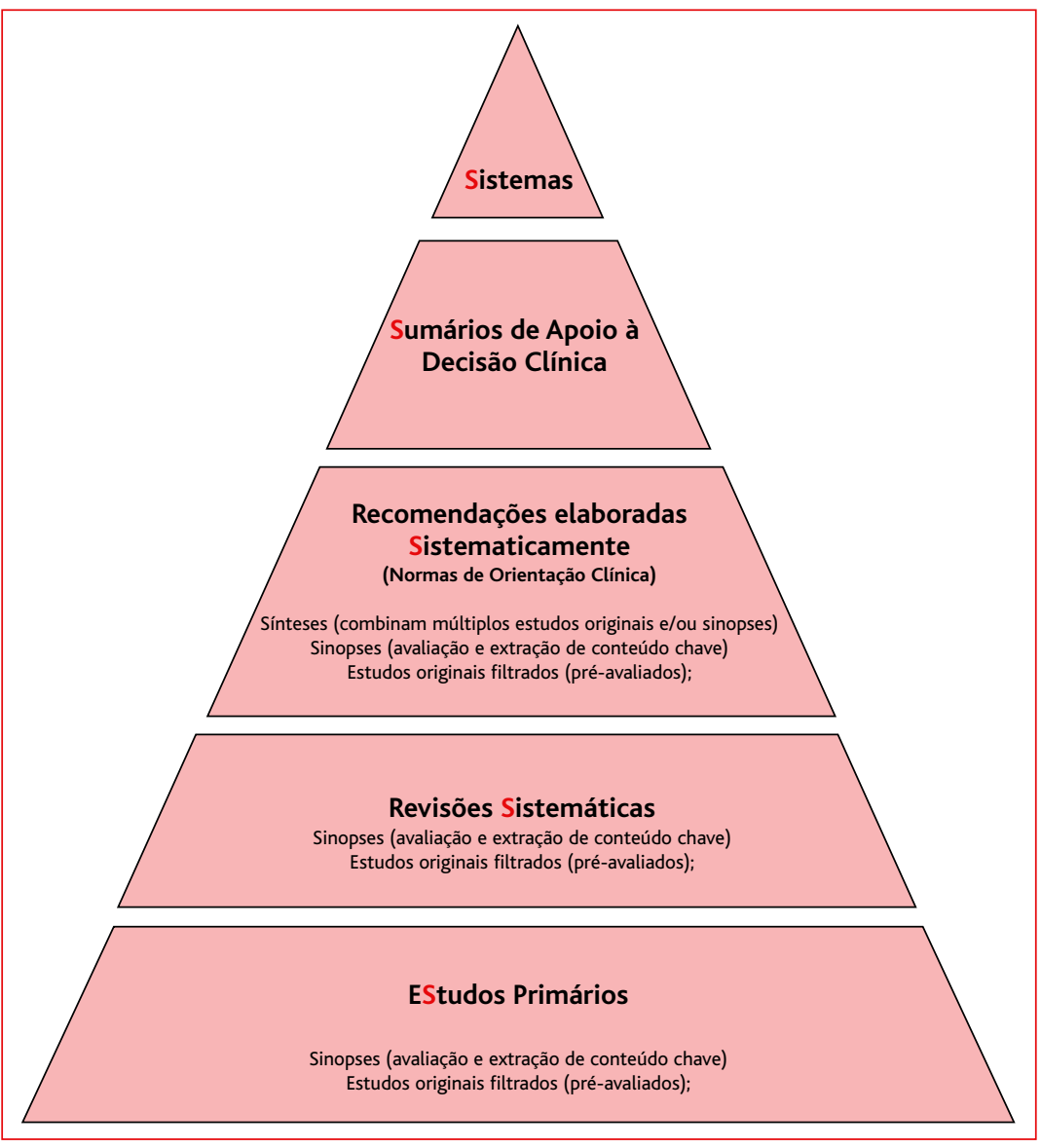

Figura 2. A pirâmide $5 S$ da prática clínica baseada na evidência. Adaptado de: Alper BS, Haynes RB. EBHC pyramid 5.0 for accessing preappraised evidence and guidance. Evid-Based Med. 2016;21(4):123-5. fornecem informação que responde tanto a perguntas de conhecimento de base como a perguntas orientadas por problemas. As ferramentas neste nível apreciam criticamente e resumem informação dos três níveis inferiores da pirâmide. Nelas encontramos conteúdos que são escritos por autores obedecendo a critérios e metodologia de síntese de evidência explícita, como por exemplo a metodologia Grading of Recommendations, Assessment, Development, and Evaluation-GRADE, ${ }^{12}$ supervisionada por um corpo editorial e com revisão entre pares (peer review). Quando a ferramenta é credível, ou seja, a sua metodologia de síntese de evidência é robusta e encontramos a resposta à nossa pergunta neste nível, não faz sentido perder tempo a procurar em níveis inferiores.

No terceiro nível da pirâmide encontramos recomendações elaboradas sistematicamente. São as de- nominadas normas de orientação clínica (guidelines, em inglês). Estas têm normalmente um foco mais específico que os sumários de apoio à decisão clínica, como uma doença (diabetes, por exemplo) ou um aspeto clínico dentro de uma doença (e.g., gestão do pé diabético). Apesar desse foco mais específico, a elaboração de recomendações deve seguir o mesmo rigor metodológico. De novo, se neste nível de informação encontramos uma resposta clara, consistente e baseada em recomendações elaboradas de forma metodologicamente adequada, pode não fazer sentido procurar em níveis inferiores. A vantagem de ficarmos por estes degraus da pirâmide de evidência é a garantia de que a evidência foi pré-avaliada e será de boa qualidade. No entanto, nova informação demora tempo a ser incorporada nos níveis superiores da pirâmide, pelo que caso 


\begin{tabular}{|c|c|}
\hline Nível & \\
\hline \multirow[t]{3}{*}{ Transversal a todos os níveis* } & TRIP Database - http://www.tripdatabase.com \\
\hline & Epistemonikos - http://www.epistemonikos.org \\
\hline & ACCESSS - http://plus.mcmaster.ca/accessss \\
\hline \multirow[t]{5}{*}{ Sumários de Apoio à Decisão Clínica } & BMJ Best Practice - https://bestpractice.bmj.com \\
\hline & Dynamed - https://www.dynamed.com \\
\hline & UpToDate - https://www.uptodate.com \\
\hline & Essential Evidence Plus - http://www.essentialevidenceplus.com \\
\hline & Medscape - https://emedicine.medscape.com \\
\hline \multirow[t]{4}{*}{ Recomendações elaboradas sistematicamente } & Guidelines GRADE - https://guidelines.gradepro.org/search \\
\hline & NICE - https://www.nice.org.uk \\
\hline & SIGN - https://www.sign.ac.uk \\
\hline & GuidelineCentral - https://www.guidelinecentral.com \\
\hline \multirow[t]{2}{*}{ Revisões sistemáticas } & Cochrane Library - https://www.cochranelibrary.com \\
\hline & CRD Database - http://www.york.ac.uk/crd/ \\
\hline \multirow[t]{4}{*}{ Estudos primários } & PubMed Clinical Queries - https://www.ncbi.nlm.nih.gov/pubmed/clinical \\
\hline & Google Scholar - https://scholar.google.com \\
\hline & Clinicaltrials.gov - https://clinicaltrials.gov \\
\hline & Registo Ensaios Clínicos OMS - https://www.who.int/ictrp/en/ \\
\hline
\end{tabular}

*Estas ferramentas permitem aplicar filtros para os diferentes níveis da pirâmide.

queiramos estar confiantes de que estamos ao corrente da informação mais recente é recomendável pesquisar nos dois níveis basais da pirâmide: as revisões sistemáticas e os estudos primários.

As revisões sistemáticas são estudos que, como o próprio nome indica, de uma forma sistemática procuram intensamente por estudos primários e aglomeram a prova científica à volta de uma pergunta clínica de forma a fornecer uma visão global dos efeitos de determinada intervenção ou fenómeno médico. ${ }^{10} \mathrm{Como}$ antes referido, a Colaboração Cochrane é uma das fontes mais credíveis para obtenção deste tipo de estudos e na sua biblioteca online encontramos inúmeras revisões sistemáticas nos mais variados tópicos médicos, ainda que com especial enfoque nas intervenções preventivas e terapêuticas.

A base da pirâmide $5 S$ é composta por estudos primários. Se todos os outros níveis não nos deram uma resposta satisfatória devemos procurar nos estudos originais, idealmente pré-avaliados ou em formato de sinopse. Uma pesquisa a este nível requer a utilização de termos MeSH e operadores boleanos, cuja correta utilização ultrapassa o âmbito deste artigo.

No Quadro III sugerimos algumas ferramentas eletrónicas referentes a cada um dos níveis da pirâmide 5S. Sugerimos começar primeiro por bases de dados que providenciam resultados de todos os níveis da pirâmide, como a TRIP Database, a Epitemonikos ou a plataforma ACCESSS da Universidade de McMaster. Estas ferramentas têm habitualmente filtros que nos permitem obter resultados de um ou vários níveis da pirâmide 5S, como por exemplo apenas normas de orientação clínica ou apenas revisões sistemáticas. $\mathrm{O}$ acesso a algumas destas plataformas pode implicar o pagamento de uma subscrição, ainda que muitas outras são de acesso gratuito. Caso haja interesse na pesquisa de ar- 
tigos primários, sugerimos a utilização da opção Clinical Queries, da PubMed, que disponibiliza filtros de pesquisa detalhados para conteúdos de terapia, diagnóstico, prognóstico, etiologia, regras de predição clínica e ainda um filtro para revisões sistemáticas. ${ }^{13}$ No entanto, desencorajamos a tentação de realizar pesquisas não estruturadas na MEDLINE para responder a dúvidas clínicas fora do âmbito de investigação clínica. Com todos os recursos que hoje em dia temos disponíveis devemos primordialmente aceder a recursos que nos ofereçam a informação pré-avaliada.

\section{PASSO 3: AVALIAR}

Depois de elaborarmos uma pergunta bem formulada e encontrarmos informação que nos permita responder à mesma, devemos avaliar criticamente a prova científica encontrada.

Como anteriormente referido, a maior parte das nossas perguntas devem ser respondidas utilizando numa fase inicial os níveis superiores da pirâmide dos 5S. Muitas das ferramentas no nível sistema e no nível sumário da pirâmide $5 \mathrm{~S}$ têm critérios transparentes de pesquisa de evidência, de apreciação crítica da qualidade metodológica dos estudos e ainda identificam os critérios usados para a elaboração de recomendações. Por isso, faz sentido que os clínicos deleguem nestas instituições o trabalho de adquirir e avaliar a qualidade metodológica de estudos primários, revisões sistemáticas e recomendações elaboradas sistematicamente. ${ }^{14-16}$

No nível das recomendações elaboradas sistematicamente os autores devem deixar explícita qual a prova científica que suporta as recomendações (idealmente revisões sistemáticas), qual a avaliação que foi feita a essa prova (e.g., «muito baixa», «baixa», «moderada», «elevada») e qual a força com que os autores classificam a recomendação, por exemplo, «forte» (a maior parte dos doentes devem receber esta recomendação) ou «condicional» (os médicos devem ajudar os doentes a decidir se a recomendação é concordante com os seus valores). ${ }^{4}$

Nos níveis mais baixos da pirâmide 5S, tanto as revisões sistemáticas como os estudos primários requerem o domínio de conceitos epidemiológicos e estatísticos que devem ajudar-nos a responder a duas perguntas: será que esta informação é válida? E será que é clinicamente importante? As duas devem ter resposta positiva para considerarmos prosseguir para o passo 4 e aplicar a informação aos nossos doentes.

Não é nossa pretensão escrever num único artigo todos os conteúdos de avaliação de literatura médica reunidos em livros de texto ou cursos presenciais de vários dias. No entanto, para avaliar a validade metodológica da maior parte dos estudos, faz sentido pensar em três perguntas genéricas cujo objetivo é detetar algum viés (entendido como um desvio sistemático da verdade) que possa invalidar os resultados reportados.

I-Qual a pergunta de investigação subjacente a este estudo?

Esta primeira pergunta remete-nos de novo para o acrónimo PICO. Será que a pergunta que justificou o estudo em análise é idêntica à nossa pergunta clínica em todos os seus componentes?

\section{II - O método usado é válido?}

Esta pergunta tenta detetar erros metodológicos na execução do estudo. ${ }^{4}$ De novo encontramos utilidade pensar na PICO para detetar vieses. Quem é a população? Como foi definida? Como foram recrutados os participantes, selecionados e alocados à intervenção ou exposição? Depois, qual a intervenção, exposição, teste ou fenómeno em estudo? Qual o comparador e como foram estes participantes escolhidos? As variáveis resultados medidas (outcomes) são importantes para os doentes? Foram medidas de forma cega e clinicamente adequada?

Interessa depois refletir sobre o desenho de estudo escolhido para responder à pergunta. É de especial relevância entender que diferentes perguntas clínicas requerem diferentes desenhos de estudo. Já vimos que a maior parte das perguntas que fazemos quando cuidamos de doentes correlacionam-se com os principais temas e tarefas dos cuidados de saúde (Quadro II): aspetos clínicos do doente ou da doença; etiologia e risco; diagnóstico diferencial; testes diagnósticos; prognóstico; tratamento; prevenção; experiência ou perceções dos doentes; melhoria de cuidados. ${ }^{17}$ Cada um dos diferentes tipos de pergunta requer um estudo com determinado desenho metodológico para adequadamente responder à pergunta (Quadro IV).

As perguntas de tratamento são aquelas que visam escolher tratamentos eficazes e que vão de encontro aos 
valores do doente (eficácia na melhoria de sintomas, funcionalidade, morbilidade, qualidade de vida e mortalidade). O desenho de estudo epidemiológico adequado para responder a este tipo de perguntas é o ensaio clínico aleatorizado. Este desenho de estudo experimental determina que, através de um processo aleatório como lançar uma moeda ao ar, os participantes no estudo sejam alocados a um braço experimental (aquele que recebe a intervenção, por exemplo, um medicamento) ou a um braço controlo (aquele que recebe o comparador, por exemplo, placebo). O processo aleatório garante que no início do estudo não há qualquer diferença entre os dois grupos e que a única diferença no final é que um dos grupos foi sujeito a uma intervenção e o outro não. Assume, portanto, que no final do estudo qualquer diferença entre os grupos se deve à intervenção.

As perguntas de etiologia procuram responder ou identificar causas de doenças e as suas vias fisiopatológicas. Neste caso, por questões éticas evidentes, não podemos experimentar sobre pessoas submetendo algumas a um potencial fator etiológico e outras não para verificar se desenvolvem ou não uma doença. Por isso, o desenho de estudo epidemiológico recomendado é um estudo observacional (caso-controlo ou coorte) no qual pessoas com e sem fatores de exposição que nos interessam são seguidas no tempo para determinar se desenvolvem, ou não, a variável resultado que estamos a estudar.

Perguntas de diagnóstico procuram valorizar a utilidade de sinais, sintomas ou testes para diagnosticar uma doença. Estes estudos têm um desenho próprio. Neles, investigadores identificam um grupo de participantes no qual existe a suspeita que uma doença está presente, aplicam o teste diagnóstico que está a ser avaliado (seja um teste analítico, uma manobra do exame objetivo ou um sinal clínico) e, de seguida, aplicam o teste de diagnóstico considerado referência (gold standard, em inglês). O objetivo é comparar o desempenho do teste diagnóstico em estudo com aquele que é considerado o melhor método diagnóstico para aquela doença.

As perguntas de prognóstico procuram avaliar a importância de fatores que podem potencialmente alterar o curso provável da doença ao longo do tempo. Estas perguntas devem ser respondidas com estudos de prognóstico. Neste tipo de estudos, os investigadores começam por identificar participantes que pertencem a um determinado grupo (como, por exemplo, diabéticos com HbAlc superior a 10\%; homens que sofreram um enfarte agudo do miocárdio nos últimos seis meses ou doentes com neoplasia da mama) com e sem fatores que possam alterar o seu prognóstico (e.g., idade, comorbilidades ou rendimento familiar). Estes participantes são depois seguidos no tempo para determinar se a doença ou evento clínico em estudo se desenvolve ou não (como, por exemplo, amputações de pé diabético, recorrência do enfarte agudo do miocárdio ou mortalidade). Os estudos de prognóstico são considerados por alguns autores estudos observacionais em que o elemento tempo é considerado a variável exposição (inception cohort, em inglês). ${ }^{4}$

Por vezes, as perguntas que fazemos sobre a prática clínica implicam uma análise económica que compara os custos e consequências das diferentes decisões. Este tipo de perguntas requer respostas provenientes de estudos de avaliação económica. São estudos de elevada complexidade na execução e apreciação crítica, mas preciosa utilidade na informação de decisões de política de saúde. Estes estudos habitualmente não incidem sobre o efeito ou custo de uma intervenção específica, mas sobre toda a cascata de custos e consequências que a adoção ou não adoção de determinada intervenção tem sobre um sistema de saúde ou organização. Aqueles que medem custo-efetividade reportam habitualmente os seus resultados em ganhos na quantidade e na qualidade de vida dos indivíduos, os Quality Adjusted Life Years (QALYs). ${ }^{18}$

Por vezes, algumas das perguntas mais relevantes que fazemos não são passíveis de medições quantitativas e requerem uma abordagem qualitativa. Perguntas sobre perceções de satisfação, significados, determinantes de comportamentos ou qualidade de vida requerem metodologias que capturem e analisem palavras, discursos, de forma a entender os fenómenos.

\section{III - Quais os resultados?}

Nesta pergunta interessam-nos resultados que permitam saber quais são as recomendações e a sua força nas normas de orientação clínica; qual é a eficácia e precisão das intervenções nos estudos quantitativos e revisões sistemáticas dos mesmos; ou quais são os principais temas nos estudos qualitativos. 
QUADRO IV. Tipos de perguntas clínicas e correspondente desenho de estudo

\begin{tabular}{l} 
Estudo - Desenho $\mid$ Aspetos importantes do desenho \\
\hline Recomendações elaboradas sistematicamente \\
\hline Definição clara das perguntas incluídas na orientação clínica, critérios claros \\
para escolher o painel que elabora recomendações (formação científica, \\
contributo dos doentes, gestão de conflitos de interesse), utilização de \\
revisões sistemáticas para sintetizar a melhor prova científica sobre cada \\
uma das perguntas, utilização de critérios claros para transformar prova \\
científica em recomendações de prática, critérios claros para determinar a \\
força de recomendação.
\end{tabular}

\section{Revisões sistemáticas}

Pesquisa bibliográfica exaustiva (>=2 bases bibliográficas, revisão das referências bibliográficas e contacto com autores), seleção e avaliação metodológica por pelo menos dois autores, processo feito de acordo com o que foi escrito no protocolo.

Resultados

Recomendações e força de recomendação.

Os resultados dependem do tipo de pergunta (ver abaixo).

Deve ser fornecida uma estimativa da consistência da literatura (i.e., se os resultados são semelhantes de estudo para estudo) e uma avaliação do potencial para viés de publicação.

\begin{tabular}{|c|c|c|}
\hline \multicolumn{3}{|l|}{ Estudos primários } \\
\hline $\begin{array}{l}\text { Tratamento - Ensaio } \\
\text { clínico }\end{array}$ & $\begin{array}{l}\text { Aleatorização; ocultação da sequência aleatória; } \\
\text { "cegueira" de participantes, clínicos e avaliadores } \\
\text { de resultados; seguimento completo; relato de } \\
\text { todos os resultados previstos no protocolo. }\end{array}$ & $\begin{array}{l}\text { Eventos binários: risco relativo e redução de risco } \\
\text { absoluto. } \\
\text { Eventos contínuos: diferença de média. }\end{array}$ \\
\hline $\begin{array}{l}\text { Etiologia - } \\
\text { Estudo de coorte ou } \\
\text { caso-controlo }\end{array}$ & $\begin{array}{l}\text { Ajuste estatístico dos resultados às principais } \\
\text { variáveis de confundimento, medição igual de } \\
\text { resultados para grupos expostos e não expostos, } \\
\text { medição igual de exposição para doentes e não } \\
\text { doentes. }\end{array}$ & Razão de possibilidades (odds ratio). \\
\hline $\begin{array}{l}\text { Diagnóstico - Estudo } \\
\text { de acuidade } \\
\text { diagnóstica }\end{array}$ & $\begin{array}{l}\text { Utilização do mesmo padrão de ouro em todos os } \\
\text { participantes, avaliação cega do novo teste e do } \\
\text { padrão de ouro. }\end{array}$ & $\begin{array}{l}\text { Testes binários: razão de verosimilhança } \\
\text { (likelihood ratio). } \\
\text { Testes contínuos: área debaixo da curva recetor } \\
\text { operador (Area under the Receiver operator curve). }\end{array}$ \\
\hline $\begin{array}{l}\text { Prognóstico - Coorte } \\
\text { de origem (inception } \\
\text { cohort) }\end{array}$ & $\begin{array}{l}\text { Amostra representativa de doentes, } \\
\text { homogeneidade dos participantes, seguimento } \\
\text { completo dos participantes. }\end{array}$ & $\begin{array}{l}\text { Curva de Kaplan Meyer. } \\
\text { Tempo de sobrevivência médio. }\end{array}$ \\
\hline $\begin{array}{l}\text { Custo-efetividade - } \\
\text { Estudo de avaliação } \\
\text { económica }\end{array}$ & $\begin{array}{l}\text { Escolha clara da perspetiva (doente, pagador ou } \\
\text { sociedade), medição clara e exaustiva dos custos, } \\
\text { transparência na escolha do modelo. }\end{array}$ & Custo-efetividade (EUR por evento). \\
\hline $\begin{array}{l}\text { Experiências de saúde } \\
\text { e doença - Estudo } \\
\text { qualitativo }\end{array}$ & $\begin{array}{l}\text { Justificação da escolha dos participantes, das } \\
\text { formas de colheita de dados e da forma de análise; } \\
\text { mecanismos adoptados pelos investigadores para } \\
\text { abordarem os seus preconceitos face ao fenómeno } \\
\text { em estudo. }\end{array}$ & Temas principais. \\
\hline
\end{tabular}




\section{PASSO 4: APLICAR}

Depois de estruturar uma pergunta clínica, adquirir e avaliar a melhor prova científica é tempo de aplicar a mesma.

\section{Da prova científica até a prática clínica}

A aplicação de prova científica será mais imediata quando utilizamos sumários ou recomendações elaboradas sistematicamente. Isto porque estes recursos habitualmente integram a prova científica e transformam-se em recomendações de prática clínica. Estas recomendações podem ser fortes, se a prova científica que as sustenta for robusta, se for razoável assumir que não existem grandes variações nas preferências dos utentes e se existe um impacto social (em termos de custo-efetividade, equidade ou outros). No fundo, recomendações fortes significam que a recomendação deve ser implementada na maioria dos doentes naquelas circunstâncias. ${ }^{19}$ Se a nossa população apresentar características muito específicas, por razões epidemiológicas ou culturais, pode ser necessária uma adaptação destas recomendações. As recomendações podem ser fracas, quando a prova científica for pouco robusta ou inconsistente, quando é expectável que a decisão seja muito sensível a diferenças nos valores e preferências dos utentes ou quando há dúvidas sobre o impacto na equidade e utilização de recursos. No fundo, recomendações fracas implicam que haja uma negociação entre o médico e o utente sobre a forma de proceder. ${ }^{19}$ De qualquer forma, a aplicação de prova científica é mais imediata nos sumários e recomendações elaboradas sistematicamente porque houve já um grupo de pessoas que procurou integrar prova científica, preferência dos doentes e experiência clínica, poupando esse trabalho a quem quer tomar decisões numa situação de urgência ou numa consulta.

\section{A experiência clínica}

A aplicação de resultados de revisões sistemáticas e estudos originais à prática clínica é mais exigente. Este tipo de estudos caracteriza o prognóstico de uma doença, os efeitos de um tratamento ou a acuidade de um teste diagnóstico com algum grau de incerteza. Os estudos raramente são perfeitos e nem sempre os resultados são tão claros como se desejaria. Sem um painel que transforme a prova científica numa recomenda- ção, caberá ao clínico avaliar se pode basear a sua decisão clínica no resultado desse estudo. Para isso devemos considerar particularidades da nossa prática ou dos nossos doentes que possam alterar a confiança que temos nas estimativas de efeito do tratamento. Deve responder-se a perguntas como se os meus doentes são comparáveis com os doentes do estudo? É possível reproduzir a intervenção estudada no meu contexto de trabalho? O comparador usado corresponde à prática clínica habitual no meu contexto de trabalho? Será que as variáveis resultado avaliadas (outcomes) correspondem ao que os doentes da minha prática mais valorizam? Será que os potenciais benefícios justificam os potenciais riscos? ${ }^{4}$

Para além destas questões, devemos saber como transmitir informação e como envolver o doente na decisão clínica.

\section{PASSO 5: ATUAR - COMO MUDAR A PRÁTICA?}

Mencionámos previamente que a MBE promove a integração da melhor prova científica com a experiência clínica e os valores e preferências dos doentes. Tal implica decisões colaborativas entre pessoas que têm ideias, expectativas e receio ${ }^{20}$ e profissionais de saúde. Ambos têm informação relevante que permite respeitar a autonomia, direito à autodeterminação e individualidade do doente. ${ }^{21}$ Estes dois princípios justificam a decisão partilhada. No entanto, a maioria dos clínicos adeptos da decisão partilhada já se confrontaram com a pergunta: "E a Dra., no meu lugar, o que faria?". Na realidade, pesar benefícios e malefícios de um tratamento pode ser uma tarefa cognitivamente muito exigente e poderá ser particularmente difícil em situações de doença, nas quais existe um componente emocional muito forte. No entanto, o envolvimento dos doentes na decisão pode ser facilitado por estratégias de transmissão de informação e de clarificação de valores.

\section{Como envolver os doentes na decisão}

A transmissão de informação é mais fácil quando a informação é personalizada e relevante, quando a comunicação integra linguagem verbal e recursos visuais e quando os números são apresentados sob a forma de frequências naturais e se mantém constante o mesmo denominador para os diferentes efeitos da interven- 
ção. ${ }^{22-24}$ Por exemplo, ao falar de rastreio do cancro da mama pode-se informar que em cada 1.000 mulheres de 50 anos, antecipamos que cinco possam vir a falecer de cancro da mama (informação personalizada). Nessas 1.000 mulheres pode haver uma vida salva por efeito da mamografia (utilização de frequências naturais). Nessas 1.000 mulheres, 100 tiveram uma alteração na mamografia que mais tarde se mostrou que não era devida a cancro e cinco sofreram uma mastectomia por uma suspeita de cancro que não se confirmou (utilização de frequências naturais, sempre com o mesmo denominador de 1.000 mulheres). Esta comunicação verbal poderia ser complementada por um pictograma.

Muitas vezes uma intervenção leva a múltiplos efeitos, uns benéficos e outros prejudiciais. Nessas circunstâncias podemos auxiliar o doente a compreender o que é mais importante para ele. Por exemplo, uma doente pondera a decisão de uma prótese total do joelho. Essa prótese reduzirá a dor e permitirá aumentar a capacidade de fazer marcha e fazer as suas atividades de vida diária num horizonte de 3-6 meses. No entanto, na fase inicial poderá ter mais dor (pós-operatória) e limitação funcional, sendo expectável que nos primeiros três meses terá de fazer reabilitação intensiva. Há ainda o risco de uma complicação da cirurgia e ficar, a longo prazo, pior da dor e da função que atualmente. Uma técnica, relativamente simples, de clarificação de valores é expor os prós e os contras de cada uma das decisões. ${ }^{25}$ Outra técnica é pedir ao doente para ordenar do mais importante para o menos importante: a dor e a função nos próximos seis meses, a dor e a função de seis meses em diante e a certeza de saber qual a dor e a função que terá daqui a seis meses. $^{26}$

Existem ferramentas, chamadas auxiliares de decisão, que são baseadas na melhor prova científica disponível e são desenhadas para ajudar os utentes a participar nas decisões de saúde e a melhorar a qualidade das decisões. Revisões sistemáticas de ensaios clínicos sugerem que os utentes se sentem com mais conhecimentos, melhor informados e mais cientes dos seus próprios valores com a utilização dos auxiliares de decisão, seja em formato papel, vídeo ou online. ${ }^{27-28}$ Estes instrumentos permitem que os utentes melhorem o seu conhecimento sobre as várias opções de tratamento, tenham expectativas mais realistas sobre os be- nefícios e malefícios dos tratamentos e, possivelmente, maior congruência entre a decisão tomada e os seus valores.

\section{A pergunta tem resposta, devo divulgar?}

A nossa filosofia é que a aprendizagem em grupo, além de mais divertida, é mais eficaz. Sugerimos, por isso, que as unidades funcionais dos cuidados primários, os departamentos hospitalares ou grupos informais de profissionais de saúde mantenham um espaço onde possam discutir as dúvidas clínicas que não foram capazes de responder e que procurem respondê-las usando esta metodologia dos 5S. No caso de necessitarem de ler recomendações elaboradas sistematicamente, revisões sistemáticas ou estudos originais, recomendamos que usem grelhas de apoio à leitura crítica. Estas grelhas procuram sinalizar qual é a informação importante a pesquisar nos métodos e resultados para saber se o estudo é credível e se os resultados podem ser aplicados à prática clínica. Abundam inúmeros exemplos na Internet de livre acesso. ${ }^{29} \mathrm{~A}$ divulgação destas perguntas e das respostas encontradas apenas faz sentido quando se cumpram critérios de relevância para a prática clínica, novidade e qualidade metodológica acompanhada da adequada apreciação crítica de todos os passos necessários à obtenção e implementação de uma resposta adequada.

\section{A pergunta não tem resposta, e agora?}

Se a pergunta que fizemos não tem resposta em nenhuma das fontes acima citadas podemos estar perante uma pergunta de investigação inovadora. Nesse caso podemos avançar para um trabalho de revisão mais aprofundado. O método de revisão mais fidedigno será uma revisão sistemática, que é um processo moroso e deve ser conduzido por uma equipa de investigadores (de preferência, pelo menos, um autor com experiência em revisões sistemáticas). Um método mais simplificado, mas ainda assim rigoroso, que tem sido proposto nos últimos anos é o da revisão restringida (restricted review), que pode permitir uma revisão da literatura mais rápida e com menos recursos..$^{30}$ Caso se pretenda responder a uma pergunta mais abrangente que permita uma visão global de determinado tópico então a opção deverá recair sobre uma revisão narrativa. $^{31}$ 


\section{CONCLUSÃO}

Para responder às inúmeras dúvidas clínicas que nos aparecem no dia-a-dia devemos começar por estruturar uma pergunta concreta que seja passível de responder. O passo seguinte passa por adquirir informação relevante descendo na pirâmide 5S, uma estrutura de cinco níveis hierárquicos de informação clínica para uma prática clínica baseada na evidência. $\mathrm{O}$ acesso à informação e recursos existentes hoje em dia recomenda aceder primordialmente a recursos que nos ofereçam a informação pré-avaliada. Depois devemos apreciar criticamente a informação recolhida para posteriormente avaliar a aplicabilidade no nosso contexto e, por fim, atuar e implementar na prática (se for caso disso). Este exercício deve ser incluído no dia-a-dia das unidades do Serviço Nacional de Saúde e, caso preencha critérios de novidade, rigor metodológico e relevância para a prática clínica, divulgado. No caso de encontrar uma pergunta que ainda não tem resposta poderemos estar perante uma pergunta original e poder-se-á avançar para uma investigação clínica mais aprofundada.

\section{AGRADECIMENTOS}

Os autores agradecem a Pascale Charondière, Raquel Braga, Miguel Melo, Sara Reina, Patrícia Reis e Adriana Rubín pelos comentários construtivos ao texto final.

\section{REFERÊNCIAS BIBLIOGRÁFICAS}

1. Melo M. Leitura crítica de artigos médicos [Critical reading of medical articles]. Rev Port Clin Geral. 2000;16(6):471-6. Portuguese

2. Glasziou P, Burls A, Gilbert R. Evidence based medicine and the medical curriculum. BMJ. 2008;337:a1253.

3. Sackett DL, Rosenberg WM, Gray JA, Haynes RB, Richardson WS. Evidence based medicine: what it is and what it isn't. BMJ. 1996;312(7023): 71-2.

4. Guyatt $G$, Rennie D, Meade MO, Cook DJ. Users' guides to the medical literature: a manual for evidence-based clinical practice. 3rd ed. New York: McGraw-Hill Education/Medical; 2014. ISBN 9780071790710

5. Djulbegovic B, Guyatt GH. Progress in evidence-based medicine: a quarter century on. Lancet. 2017;390(10092):415-23.

6. Bastian H, Glasziou P, Chalmers I. Seventy-five trials and eleven systematic reviews a day: how will we ever keep up? PLoS Med. 2010;7(9): e1000326.

7. Del Fiol G, Workman TE, Gorman PN. Clinical questions raised by clinicians at the point of care: a systematic review. JAMA Intern Med. 2014;174(5):710-8.

8. Osheroff JA, Forsythe DE, Buchanan BG, Bankowitz RA, Blumenfeld BH, Miller RA. Physicians' information needs: analysis of questions posed during clinical teaching. Ann Intern Med. 1991;114(7):576-81.
9. Eve R. PUNs and DENs: discovering learning needs in general practice. Oxon: CRC Press; 2003. ISBN 9781857758078

10. Straus SE, Glaziou P, Richardson WS, Haynes RB. Evidence-based medicine: how to practice and teach EBM. 5th ed. Edinburgh: Elsevier; 2018. ISBN 9780702062964

11. Alper BS, Haynes RB. EBHC pyramid 5.0 for accessing preappraised evidence and guidance. Evid Based Med. 2016;21(4):123-5.

12. Guyatt GH, Oxman AD, Vist GE, Kunz R, Falck-Ytter Y, Alonso-Coello P, et al. GRADE: an emerging consensus on rating quality of evidence and strength of recommendations. BMJ. 2008;336(7650):924-6.

13. PubMed help: NCBI manual help [Internet]. Bethesda: National Center for Biotechnology Information; 2018 [cited 2019 Jan 23]. Available from: https://www.ncbi.nlm.nih.gov/books/NBK3827/

14. Prorok JC, Iserman EC, Wilczynski NL, Haynes RB. The quality, breadth, and timeliness of content updating vary substantially for 10 online medical texts: an analytic survey. J Clin Epidemiol. 2012;65(12):1289-95.

15. Banzi R, Cinquini M, Liberati A, Moschetti I, Pecoraro V, Tagliabue L, et al. Speed of updating online evidence based point of care summaries: prospective cohort analysis. BMJ. 2011;343:d5856.

16. Kwag KH, González-Lorenzo M, Banzi R, Bonovas S, Moja L. Providing doctors with high-quality information: an updated evaluation of webbased point-of-care information summaries. J Med Internet Res. 2016;18(1):e15.

17. Straus S, Eisinga A, Sackett D. What drove the evidence cart? Bringing the library to the bedside. J R Soc Med. 2016;109(6):241-7.

18. Drummond MF, Jefferson TO. Guidelines for authors and peer reviewers of economic submissions to the BMJ: the BMJ Economic Evaluation Working Party. BMJ. 1996;313(7052):275-83.

19. Guyatt GH, Oxman AD, Kunz R, Falck-YtterY, Vist GE, LiberatiA, et al. Going from evidence to recommendations. BMJ. 2008;336(7652):1049-51.

20. Nunes JM. Comunicação em contexto clínico. Lisboa: Bayer Health Care; 2010.

21. Elwyn G, Frosch D, Thomson R, Joseph-Williams N, Lloyd A, Kinnersley $P$, et al. Shared decision making: a model for clinical practice. J Gen Intern Med. 2012;27(10):1361-7.

22. Rodrigues R, Maria AR, Bragança A, Simões S, Tomé A, Rodrigues D, et al. Comunicação e percepção de risco: diferentes modos de comunicar, diferentes modos de partilhar a decisão clínica [Risk communication and risk perception: different ways of communicating, different ways of sharing clinical decisions]. Rev Port Med Geral Fam. 2015;31(2):12533. Portuguese

23. Edwards AG, Naik G, Ahmed H, Elwyn GJ, Pickles T, Hood K, et al. Personalised risk communication for informed decision making about taking screening tests. Cochrane Database Syst Rev. 2013;(2):CD001865.

24. Akl EA, Oxman AD, Herrin J, Vist GE, Terrenato I, Sperati F, et al. Using alternative statistical formats for presenting risks and risk reductions. Cochrane Database Syst Rev. 2011;(3):CD006776.

25. Sawka CA, Goel V, Mahut CA, Taylor GA, Thiel EC, O'Connor AM, et al. Development of a patient decision aid for choice of surgical treatment for breast cancer. Health Expect. 1998;1(1):23-36.

26. Sheridan SL, Griffith JM, Behrend L, Gizlice Z, Jianwen Cai, Pignone MP. Effect of adding a values clarification exercise to a decision aid on heart disease prevention: a randomized trial. Med Decis Making. 2010;30(4): E28-39. 
27. Stacey D, Légaré F, Lewis K, Barry MJ, Bennett CL, Eden KB, et al. Decision aids for people facing health treatment or screening decisions. Cochrane Database Syst Rev. 2017;4:CD001431.

28. Baptista S, Teles Sampaio E, Heleno B, Azevedo LF, Martins C. Web-based versus usual care and other formats of decision aids to support prostate cancer screening decisions: systematic review and meta-analysis. J Med Internet Res. 2018;20(6):e228.

29. Rodrigues DS, Pinto D. Evidentia médica: avaliação e síntese de literatura médica [homepage]. Evidencia Médica.com; 2019 [cited 2019 Feb 24]. Available from: http://evidentiamedica.com

30. Plüddemann A, Aronson JK, Onakpoya I, Heneghan C, Mahtani KR. Redefining rapid reviews: a flexible framework for restricted systematic reviews. BMJ Evid Based Med. 2018;23(6):201-3.

31. Peterson J, Pearce PF, Ferguson LA, Langford CA. Understanding scoping reviews: definition, purpose, and process. J Am Assoc Nurse Pract. 2017;29(1):12-6.

32. Teeling-Smith G, Wells NE. Medicines for the year 2000: symposium proceedings. London: Office of Health Economics; 1979. ISBN 9780901387868

33. Cochrane. Cochrane collaboration: about us [homepage]. Cochrane.org; 2018 [cited 2018 May 1]. Available from: https://www.cochrane.org/ about-us

34. Grant MJ, Booth A. A typology of reviews: an analysis of 14 review ty- pes and associated methodologies. Health Info Libr J. 2009;26(2):91108.

35. Moher D, Liberati A, Tetzlaff J, Altman DG, PRISMA Group. Preferred reporting items for systematic reviews and meta-analyses: the PRISMA statement. PLoS Med. 2009;6(7):e1000097.

36. Braga R, Melo M. Como fazer uma revisão baseada na evidência [How to write an evidence based review]. Rev Port Med Geral Fam. 2009;25 (6):660-6. Portuguese

\section{CONFLITOS DE INTERESSE}

Financeiros: nada a declarar. Intelectuais: DSR, BH e CVD são coordenadores e formadores de um curso pós-graduado de avaliação de literatura médica. DSR é coordenador de uma página da internet dedicada à divulgação de literatura médica.

\section{ENDEREÇO PARA CORRESPONDÊNCIA \\ David Rodrigues \\ E-mail: dmsrodrigues@gmail.com \\ http://orcid.org/0000-0001-6785-1510}

Recebido em 26-02-2019

Aceite para publicação em 09-04-2019

\section{ABSTRACT}

\section{HOW TO ANSWER TO A CLINICAL QUESTION}

Medical knowledge is in permanent construction and being updated is an inherent task of medical practice. The purpose of this article is to review the methodology for answering clinical questions and to propose the 5A model for this purpose. Obtaining meaningful answers to clinical questions consists of adequately elaborating the question, acquiring the necessary information, critically assessing this information, applying the evidence to our context and practice, and ultimately acting to ensure that patients receive the best medical care. This exercise should be included in the day-to-day practice of medical care and, if it meets criteria of novelty, methodological rigor and relevance to clinical practice, disclosed and published.

Keywords: Evidence-based medicine; Clinical questions; Knowledge translation; Decision making; Shared informed decision; Critical appraisal. 